\title{
Screening of significantly hypermethylated genes in breast cancer using microarray-based methylated-CpG island recovery assay and identification of their expression levels
}

\author{
ZHEN-QIANG LIAN, QI WANG, WEN-PING LI, AN-QIN ZHANG and LING WU \\ Breast Disease Center, Guangdong Women and Children Hospital of Guangzhou \\ Medical College, Guangzhou 510010, P.R. China
}

Received February 10, 2012; Accepted April 17, 2012

DOI: $10.3892 /$ ijo.2012.1464

\begin{abstract}
To screen candidate methylation markers for early detection of breast cancer and to explore the relationship between methylation and gene expression, we performed methylated-CpG island recovery assay (MIRA) combined with CpG island array on $61982 \mathrm{CpG}$ sites across 4162 genes in 10 cancerous and 10 non-cancerous breast tissues. Direct bisulfite sequencing and combined bisulfite restriction analysis (COBRA) were carried out in independent cancerous and non-cancerous samples. Gene expression was analyzed by microarrays and validated using RT-PCR. We detected 70 significantly hypermethylated genes in breast cancer tissues, including many novel hypermethylated genes such as ITGA4, NFIX, OTX2 and FGF12. Direct bisulfite sequencing showed widespread methylation occurring in intragenic regions of the WT1, PAX6 and ITGA4 genes and in the promoter region of the OTX2 gene in breast cancer tissues. COBRA assay confirmed that the $W T 1, O T X 2$ and $P A X 6$ genes were hypermethylated in breast cancer tissues. Clustering analysis of the gene expression of 70 significantly hypermethylated genes revealed that most hypermethylated genes in breast cancer were not expressed in breast tissues. RT-PCR assay confirmed that WT1 and PITX2 were only weakly expressed in the breast cancer tissues and were not expressed in most non-cancerous breast tissues. OTX2 and PAX6 were not expressed in either breast cancer or noncancerous tissues. In conclusion, these results will expand our knowledge of hypermethylated genes and methylation sites for early detection of breast cancer and deepen our understanding
\end{abstract}

Correspondence to: Professor Qi Wang, Breast Disease Center, Guangdong Women and Children Hospital of Guangzhou Medical College, Guangzhou, Guangdong 510010, P.R. China

E-mail: wangqigz@hotmail.com

Abbreviations: COBRA, combined bisulfite restriction analysis; RT-PCR, reverse transcription PCR; MIRA, methylated-CpG island recovery assay

Key words: breast cancer, $\mathrm{CpG}$ island methylation, Methyl-CpG binding protein, microarray, gene expression, combined bisulfite restriction analysis of the relationship between methylation and gene expression. The MIRA approach can screen candidate methylated genes for further clinical validation more effectively than gene expression microarray-based strategy.

\section{Introduction}

With an estimated 1.38 million new cancer cases worldwide in 2008 , breast cancer is the most frequent cancer and the leading cause of cancer mortality in women (1). If breast cancer is detected and treated at an early stage, the majority of the patients survive. These facts highlight an urgent need to develop novel molecular biomarkers that can detect early stage of breast cancer so that treatment is initiated promptly.

Carcinogenesis has been demonstrated to involve not only genetic but also epigenetic alterations that may suppress or activate multiple genes. The most common epigenetic alteration in cancer is DNA methylation (2), which is an early event in carcinogenesis, and may therefore serve as a biomarker for the early detection of cancer. Promoter methylation, with a methyl group to the 5-carbon position of cytosine in $\mathrm{CpG}$ islands, is associated with transcriptional silencing. DNA methylation also occurs in the intragenic region. To date, little attention has been paid to intragenic methylation.

Recently, aberrant methylations of specific genes that involve breast carcinogenesis have been widely explored and the list of aberrant methylated genes is increasing $(3,4)$. Due to the limitation of techniques for analysis of DNA methylation, the majority of these efforts have focused only on individual candidate genes, which have already been identified to play a role in other cancer models. These genes are mostly tumor suppressor genes. Thus far, most methylated genes that contribute to breast cancer, such as methylated $p 16, C C D N 2$, $M G M T$ and $h M T L H l$ are identified by the candidate approach, confining to promoter methylation of tumor suppressor genes (5). This approach is not only time-consuming and labor-intensive, but also limited by candidate methylated genes and candidate methylated sites available.

Gene expression microarray based strategy has been developed for high-throughput screening of DNA methylation biomarker on a genomic scale in breast cancer. This approach uses microarray to interrogate upregulated genes 
after treatment of breast cancer line with a demethylating agent 5-aza-2'-deoxycytidine (6), and then uses bisulfite sequencing, restriction analysis, and/or methylation specific PCR to confirm DNA methylation. This approach might leave out non-expressed genes in the target tissue, thereby missing candidate genes, which may actually be potential methylation biomarkers in cancer diagnosis. For example, the significantly methylated vimentin gene has been used as a diagnostic biomarker in colorectal cancer, while colon cancer cells do not express vimentin $(7,8)$. Using the gene expression microarray based strategy, methylation of vimentin could not be identified. However, it is unclear whether there are that many such nonexpressed methylated genes in cancerous and non-cancerous tissue that critical methylation biomarkers could not be identified by gene expression microarray based strategy.

Recently, methyl-DNA immunoprecipitation combined with high-throughput sequencing (9) and methylated-CpG island recovery assay (MIRA) combined with $\mathrm{CpG}$ island array $(10,11)$ were also used to screen cancer-associated methylated genes in clinical samples of breast cancer tissue. To screen novel biomarkers for the early detection of breast cancer, in the current study, we utilized a high-throughput genome-wide approach, MIRA microarray, that is based on the high affinity of the MBD protein for methylated DNA to identify novel tumor-specific genes with promoter methylation or intragenic methylation. We also integrated the corresponding gene expression profile to explore the role of methylation in regulating gene expression.

\section{Materials and methods}

Patient samples. Samples of breast cancer tissue were obtained from the surgical specimens of 15 patients with early stage invasive breast cancer. The tissue sections were stained with $\mathrm{H} \& \mathrm{E}$ and were reviewed by a pathologist to confirm the presence of the cancer lesions. Breast cancer was staged according to the American Joint Committee on Cancer staging system protocol. ER, PR and HER2 status were determined by immunohistochemistry. Non-cancerous breast tissues were collected from the biopsy specimens of 15 patients for the suspicion of malignant lesion but without any histological findings. Clinicopathological characteristics of the patient samples are given in Table I. Ten cancerous and 10 non-cancerous breast tissues were selected for microarray analysis and the other 5 cancerous and 5 non-cancerous breast tissues were used for validation of the genes. All samples were snap-frozen in liquid nitrogen and stored at $-80^{\circ} \mathrm{C}$ until nucleic acid extraction. The study was approved by the Ethics Committee of our hospital, and all patients provided written informed consent.

MIRA microarray analysis of DNA methylation. The MIRA microarray analysis was carried out as described by Rauch et al with minor modifications (12). The schematic diagram for our MIRA-assisted $\mathrm{CpG}$ island microarray analysis is outlined in Fig. 1. Briefly, DNA was firstly separated from 10 fresh frozen cancerous and 10 non-cancerous breast tissues using standard techniques and DNA quality was verified using agarose gel electrophoresis. Then, $2 \mu \mathrm{g}$ of genomic DNA samples were digested by Mse I (5'-TTAA), which generally cuts outside of the $\mathrm{CpG}$ islands and produces
Table I. Clinicopathological characteristics of the patient samples.

\begin{tabular}{|c|c|c|}
\hline Characteristics & $\begin{array}{l}\text { Breast cancer } \\
\text { tissues }(n=15)\end{array}$ & $\begin{array}{l}\text { Breast non-cancer } \\
\text { tissues }(n=15)\end{array}$ \\
\hline Mean age (y) (range) & $41(34-50)$ & $38(32-47)$ \\
\hline Histological type & $\begin{array}{c}\text { Invasive ductal } \\
\text { carcinoma }\end{array}$ & $\begin{array}{l}\text { No histological } \\
\text { findings }\end{array}$ \\
\hline Grade $(\%)$ & & ND \\
\hline I & $5(33.3 \%)$ & \\
\hline II & $10(66.7 \%)$ & \\
\hline Cancer size $(\%)$ & & ND \\
\hline pT1 & $8(53.3 \%)$ & \\
\hline pT2 & $7(46.7 \%)$ & \\
\hline Lymph node status (\%) & & ND \\
\hline Negative & $15(100.0 \%)$ & \\
\hline Positive & $0(0.0 \%)$ & \\
\hline Estrogen receptor $(\%)$ & & ND \\
\hline Positive & $8(53.3 \%)$ & \\
\hline Negative & $7(46.7 \%)$ & \\
\hline Progesterone receptor $(\%)$ & & ND \\
\hline Positive & $9(53.3 \%)$ & \\
\hline Negative & $6(46.7 \%)$ & \\
\hline HER2 status (\%) & & ND \\
\hline$(-) /(+)$ & $10(66.7 \%)$ & \\
\hline$(++)$ & $3(20.0 \%)$ & \\
\hline$(+++)$ & $2(13.3 \%)$ & \\
\hline
\end{tabular}

200-1000 bp fragments. Fragment sizes were verified by gel electrophoresis in $1.5 \%$ agarose gels. To remove fragments smaller than $100 \mathrm{bp}$, the fragmented DNA was purified using the MicroDNA Purification Kit (Beijing CoWin Biotech Co. Ltd, Beijing, China) following the manufacturer's instructions. Subsequently, the purified DNA fragments were used to enrich methylated DNA using the BioChain MBD kit (BioChain, Hayward, CA, USA) according to the manufacturer's protocol. This kit uses the high affinity of the MBD protein for doublestranded CpG-methylation DNA. This MBD protein can bind methylated DNA fragments more sensitively and specifically than antibody, and thus has a low false positive rate in the enrichment of methylated DNA fragments (12). In addition, its procedure is simple since MBDs can bind dsDNA (12), natural status of genomic DNA, while antibody can bind only ssDNA and therefore has to denature the dsDNA before DNA enrichment in antibody based methods.

The MIRA-captured DNA segments were purified using the MicroDNA Purification Kit (CoWin Biotech Co.). After purification, the MIRA-captured DNA segments were amplified using the GenomePlex Whole Genome Amplification Kit (Sigma, St. Louis, MO, USA), according to the manufacturer's instructions. Compared with the previously reported MIRA method, in which MIRA-captured methylated DNA was added with oligonucleotide linkers for subsequent PCR-based amplification (12), the method we emloyed adopted a whole genome amplification strategy and had a few advantages, including better liner amplification, less bias in DNA amplification and did not require adding linker in amplifying MIRA-captured DNA segments. 


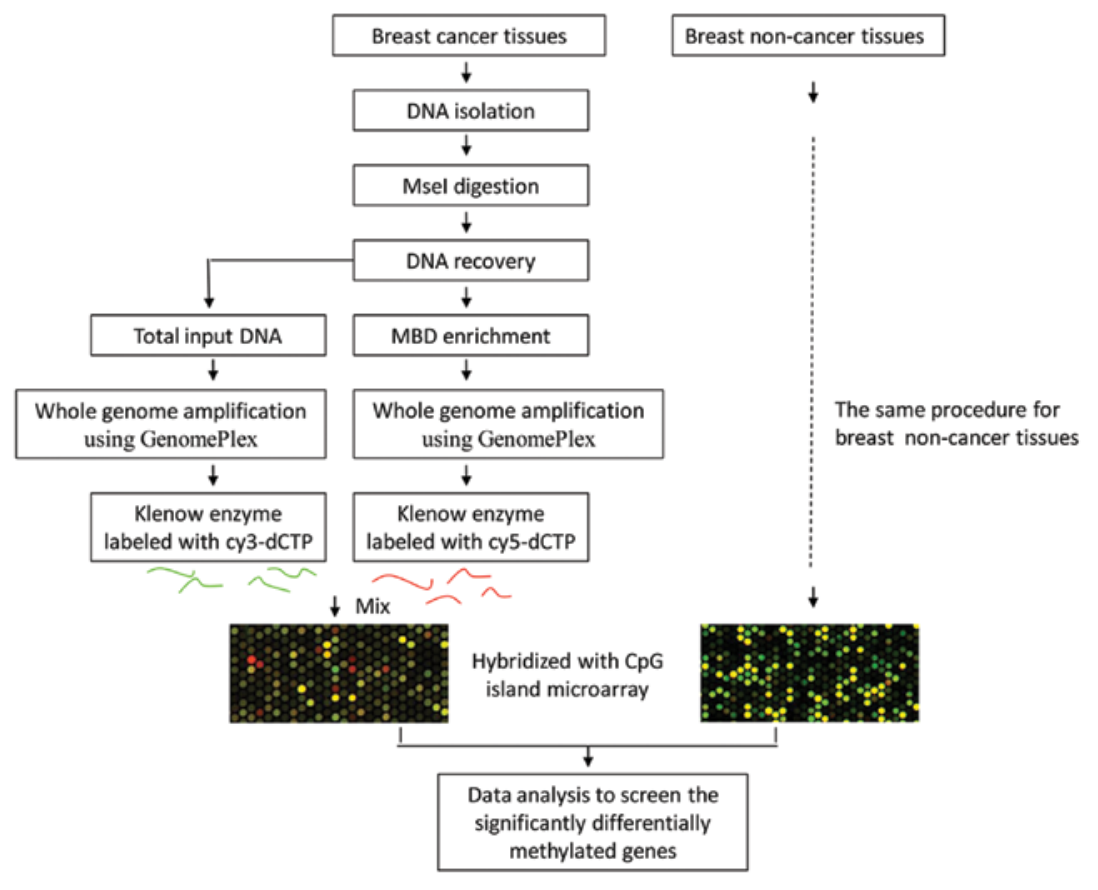

Figure 1. Schematic diagram for MIRA-assisted CpG island microarray in this study.

The products of whole genome amplification from the total input DNA without MBD enrichment and methylationenriched DNA from each sample were labeled with cy3-dCTP and cy5-dCTP using Klenow enzyme (Takara, Dalian, China), respectively. Prior to labeling with fluorescent dye, DNA amplification products were purified using the MicroDNA Purification Kit. The fluorescent dye labeled DNA segments were then pooled and hybridized to Agilent human $\mathrm{CpG}$ island microarrays, which were designed to interrogate $61982 \mathrm{CpG}$ dinucleotides, covering 4162 genes. Following hybridization, washing and scanning were carried out on Agilent's microarray platform according to Agilent's standard protocols. Agilent Feature Extraction software was used to extract the hybridization signals from microarrays. Following global mean normalization, probes with an intensity $<400$ were discarded from further analysis.

It is critical to define a proper cut-off value in fold change in MIRA/input signaling ratios between cancer versus non-cancer to efficiently screen candidate hypermethylated genes. However there are still no gold standards on setting up fold change cutoff for defining candidate methylated genes. In fact, methylation difference in the MIRA approach is represented by copy number difference of DNA enriched by MBD protein. It is similar to DNA copy number difference in array-based comparative genomic hybridization $(\mathrm{aCGH})$ and quite different from gene expression difference in gene expression microarray analysis, for one copy of DNA expresses various copies of mRNA. In well-established aCGH, cutoff for detecting DNA copy difference is usually defined as probe ratio value $<1.25(13,14)$, which is smaller than that for mRNA detection. Therefore, probes with more than 1.2 fold changes $(\mathrm{p}<0.01)$ in MIRA/input signaling ratios between cancer versus non-cancer were selected here as positive probes using SAM software and genes with at least two posi- tive probes were defined to be significantly hypermethylated genes. Hypermethylated genes were further analyzed based on a significant enrichment of GO terms using hyper-geometric distribution in the $\mathrm{R}$ language software package.

Gene expression microarray analysis. RNA from 8 of the 10 cancerous and 8 of the 10 non-cancerous breast tissues subjected to MIRA microarray analysis were qualified and interrogated for gene expression profiling. Briefly, total RNA was extracted using TRIzol reagent (Invitrogen Life Technolgies, Carlsbad, CA, USA). mRNA expression levels were measured using Agilent Human Whole Genome $8 * 60 \mathrm{~K}$ array following the manufacturer's protocol. Arrays were washed and scanned on Agilent's microarray platform according to Agilent's standard protocols. SAM software was used to analyze differentially expressed genes between cancerous and non-cancerous breast tissues.

Validation of hypermethylated genes. To validate the reliability of MIRA microarray data and to explore the methylation extent of hypermethylated genes identified by methylation microarray analysis, 4 significantly hypermethylated genes identified by methylation microarray analysis were selected for further validation using direct bisulfite sequencing. To validate the reliability of MIRA microarray data and explore the relationship between methylation and gene expression, 4 significantly hypermethylated genes were selected based on methylation and gene expression microarray data for further validation in 5 independent cancerous and 5 independent non-cancerous samples using combined bisulfite restriction analysis (COBRA) (15). For PCR amplification, direct bisulfite sequencing and COBRA assay used the same PCR reaction system, including the same primers that are specific for the bisulfite modified DNA and contain minimum $\mathrm{CpG}$ sites in 
Table II. Primer pairs for COBRA validation of hypermethylated genes.

\begin{tabular}{lll}
\hline Gene & GenBank no. & \multicolumn{1}{c}{ Primer pairs (5'-3') } \\
\hline WT1 & NM_024426.4 & F: AAAATTATTCGAGGGTTTAGGG \\
OTX2 & R: TCTACTTACGATTCCTCCACAA \\
PAX6 & F: AGTTGTGTTAGGTTGAGGGAG \\
& & R: AATCCCAAAAACCTTTTAAA \\
PITX2 & NM_000280.3 & F: AAAAGGAAGTTAGGAGAAATYGGAA \\
& NM_000325.5 & F: AAATCACTCAAACGAAATTAAACTACAC \\
& & R: CCACTACATACTAACAAACACTCAAAT \\
\hline
\end{tabular}

F, forward; R, reverse.

Table III. Primer pairs for RT-PCR validation of expression levels of hypermethylated genes.

\begin{tabular}{lll}
\hline Gene & Genbank No & \multicolumn{1}{c}{ Primer pairs $\left(5^{\prime}-3^{\prime}\right)$} \\
\hline WT1 & NM_024426.4 & $\begin{array}{l}\text { F:CATACCAGTGTGACTTCAAGGA } \\
\text { R: ATGTTGTGATGGCGGACTAAT }\end{array}$ \\
OTX2 & NM_172337.1 & F: CCAGACATCTTCATGCGAG \\
PAX6 & NM_000280.3 GTCCACTCTCTGAACTCACT \\
PITX2 & FM_000325.5 & F: CCCAGCCAGACCTCCTCATA \\
& & F: CCGGAACTTGAACTGGAAC \\
& & R: CGGCCCACGGAGTTGTTGTAGGAA \\
\hline
\end{tabular}

F, forward; R, reverse.

their sequence (Table II). First, genomic DNA was subjected to bisulfite treatment using the DNA methylation detection kit (BioChain). Then, $125 \mathrm{ng}$ of sodium bisulfite-treated DNA was used for a program of 45 cycles of melting (30 sec at $\left.94^{\circ} \mathrm{C}\right)$, annealing $\left(30 \mathrm{sec}\right.$ at $\left.55^{\circ} \mathrm{C}\right)$ and extension $(30 \mathrm{sec}$ at $72^{\circ} \mathrm{C}$ ) in a $50 \mu 1$ reaction mixture containing $1 \mathrm{X}$ PCR buffer ( $\mathrm{Mg}^{2+}$ Plus), $200 \mu \mathrm{M}$ of each $\mathrm{dNTP}, 0.5 \mu \mathrm{M}$ of forward primer, $0.5 \mu \mathrm{M}$ of reverse primer (primer sequences are provided in Table I) and 1.25 unit of Takara Taq HS. PCR products were then purified using the MicroDNA Purification Kit. For bisulfite sequencing, the purified PCR products were subjected to sequencing directly using reverse or forward primers of PCR amplification. For the COBRA assay, the purified PCR products were subjected for digestion with BstU I (CG $\downarrow C G)$ restriction enzyme (New England Biolabs, Ipswich, MA, USA) and then separated on $2 \%$ agarose gels supplemented with ethidium bromide. DNA was visualized under a UV light.

Gene expression validation of the hypermethylated genes. To explore the relationship between methylation and gene expression, the above 4 significantly hypermethylated genes that were subjected to COBRA validation were selected to validate their expression levels using reverse transcription PCR. Briefly, $1 \mu \mathrm{g}$ of DNase-treated total RNAs were reverse transcribed with oligo(dT) 15 using M-MLV reverse transcriptase (Life Technologies) in a total volume of $20 \mu 1$ reaction volume. Following reverse transcription reaction, $1 \mu \mathrm{l}$ of this mixture was employed for an RT-PCR program of 45 cycles of melting ( $30 \mathrm{sec}$ at $\left.94^{\circ} \mathrm{C}\right)$, annealing $\left(3 \mathrm{sec}\right.$ at $\left.58^{\circ} \mathrm{C}\right)$ and extension $\left(30 \mathrm{sec}\right.$ at $\left.72^{\circ} \mathrm{C}\right)$. The $20 \mu \mathrm{l}$ reaction mixture contained 1X PCR buffer ( $\mathrm{Mg}^{2+}$ Plus), $200 \mu \mathrm{M}$ of each dNTP, $0.5 \mu \mathrm{M}$ of forward primer, $0.5 \mu \mathrm{M}$ of reverse primer (Table III). Total RNA used for positive control of WT1 and PITX2 was isolated from uterine tissue and breast cancer tissue, respectively. Total RNA used for positive control of OTX2 and PAX6 was from cerebellum tissue. Glyceraldehyde 3-phosphate dehydrogenase (GAPDH) was used as internal control. RT-PCR product was separated on $2 \%$ agarose gels supplemented with ethidium bromide. DNA was visualized under a UV light.

\section{Results}

MIRA microarray analysis of DNA methylation. To screen novel methylation biomarkers for the early detection of breast cancer, we utilized the MIRA approach to identify significantly hypermethylated genes in breast cancer. Probes in which fold changes in MIRA/input signaling ratios between cancer versus non-cancer were above $1.2(\mathrm{p}<0.01)$ were defined as positive probes, and genes with at least two positive probes were defined as significantly hypermethylated genes. As a result, a total of 70 hypermethylated genes were identified (Table IV). Among them, many genes, such as ITGA4, NFIX and $F G F 12$, were first identified to be hypermethylated in the breast cancer model. 
Table IV. Hypermethylated genes identified by MIRA based microarray.

\begin{tabular}{|c|c|c|c|c|}
\hline Gene & $\begin{array}{c}\text { Changed } \\
\text { probes }\end{array}$ & $\begin{array}{c}\text { Average } \\
\text { ratio }\end{array}$ & Position & Gene description \\
\hline$C C N D 2$ & 8 & 1.70 & $\mathrm{P}$ and $\mathrm{I}$ & Cyclin D2 \\
\hline PAX6 & 8 & 2.09 & $\mathrm{P}$ and $\mathrm{I}$ & Paired box 6 \\
\hline$M N X 1$ & 6 & 2.15 & I and D & Motor neuron and pancreas homeobox 1 \\
\hline OTX2 & 6 & 1.63 & $\mathrm{P}$ and $\mathrm{D}$ & Orthodenticle homeobox 2 \\
\hline PITX2 & 6 & 1.49 & $\mathrm{P}$ and $\mathrm{I}$ & Paired-like homeodomain 2 \\
\hline NFIX & 5 & 1.66 & I & Nuclear factor I/X \\
\hline PDGFRA & 5 & 1.33 & $\mathrm{P}$ and $\mathrm{I}$ & Platelet-derived growth factor receptor \\
\hline$W T 1$ & 5 & 1.63 & I & Wilms tumor 1 \\
\hline$F G F 12$ & 4 & 2.06 & I & Fibroblast growth factor 12 \\
\hline OSRl & 4 & 1.82 & I & Odd-skipped related 1 \\
\hline SOX9 & 4 & 1.35 & $\mathrm{P}$ and $\mathrm{I}$ & Sex determining region Y-box 9 \\
\hline$V S X 1$ & 4 & 1.76 & $\mathrm{P}$ & Visual system homeobox 1 \\
\hline ATOH1 & 3 & 1.68 & $\mathrm{P}, \mathrm{I}$ and $\mathrm{D}$ & Atonal homolog 1 \\
\hline$F O X F 2$ & 3 & 1.33 & $\mathrm{P}$ & Forkhead box F2 \\
\hline$G A D 1$ & 3 & 1.48 & $\mathrm{P}$ and $\mathrm{I}$ & Glutamate decarboxylase 1 \\
\hline GATA6 & 3 & 1.62 & $\mathrm{P}$ & GATA binding protein 6 \\
\hline$G B X 2$ & 3 & 1.55 & $\mathrm{P}$ and $\mathrm{I}$ & Gastrulation brain homeobox 2 \\
\hline HAND1 & 3 & 1.52 & $\mathrm{P}, \mathrm{I}$ and $\mathrm{D}$ & Heart and neural crest derivatives expressed 1 \\
\hline$H L X$ & 3 & 1.48 & $\mathrm{D}$ & H2.0-like homeobox \\
\hline$H O X D 3$ & 3 & 1.42 & $\mathrm{P}$ and $\mathrm{I}$ & Homeobox D3 \\
\hline LHX2 & 3 & 1.63 & I & LIM homeobox 2 \\
\hline$M M P 9$ & 3 & 1.40 & I & Matrix metallopeptidase 9 \\
\hline NEUROG3 & 3 & 1.53 & $\mathrm{P}$ and $\mathrm{D}$ & Neurogenin 3 \\
\hline NID2 & 3 & 2.39 & I & Nidogen 2 \\
\hline PCDHGC5 & 3 & 2.21 & $\mathrm{D}$ & Protocadherin gamma subfamily $\mathrm{C}$ \\
\hline PDX1 & 3 & 1.85 & $\mathrm{P}$ & Pancreatic and duodenal homeobox 1 \\
\hline SIX1 & 3 & 1.37 & $\mathrm{P}$ and $\mathrm{D}$ & SIX homeobox 1 \\
\hline$T S C 2$ & 3 & 1.26 & I & Tuberous sclerosis 2 \\
\hline ACSL6 & 2 & 1.39 & $\mathrm{D}$ & $\begin{array}{l}\text { Acyl-coa synthetase long-chain family } \\
\text { member } 6\end{array}$ \\
\hline$B D N F$ & 2 & 1.45 & $\mathrm{P}$ and $\mathrm{I}$ & Brain-derived neurotrophic factor \\
\hline CACNAIE & 2 & 1.42 & I & Calcium channel, R type \\
\hline CACNAIG & 2 & 1.64 & $\mathrm{P}$ and $\mathrm{I}$ & Calcium channel, T type \\
\hline CACNAIH & 2 & 1.56 & I & Calcium channel, T type, \\
\hline$C D 8 A$ & 2 & 1.57 & I & CD8a molecule \\
\hline$C D K 6$ & 2 & 1.52 & $\mathrm{P}$ and $\mathrm{I}$ & Cyclin-dependent kinase 6 \\
\hline$C D K N 2 A$ & 2 & 1.57 & I & Cyclin-dependent kinase inhibitor $2 \mathrm{~A}$ \\
\hline CXCL12 & 2 & 1.40 & $\mathrm{P}$ & Chemokine (C-X-C motif) ligand 12 \\
\hline$D A D 1$ & 2 & 1.63 & I & Defender against cell death 1 \\
\hline$D A P K 3$ & 2 & 1.44 & $\mathrm{P}$ & Death-associated protein kinase 3 \\
\hline$D L L 4$ & 2 & 1.26 & $\mathrm{P}$ and $\mathrm{I}$ & Delta-like 4 (Drosophila) \\
\hline$E A F 1$ & 2 & 1.41 & I & ELL associated factor 1 \\
\hline HOXА & 2 & 1.36 & $\mathrm{P}$ & Homeobox A6 \\
\hline$H O X B 13$ & 2 & 2.38 & $\mathrm{P}$ and $\mathrm{I}$ & Homeobox B13 \\
\hline HOXD9 & 2 & 1.37 & $\mathrm{P}$ and $\mathrm{I}$ & Homeobox D9 \\
\hline ITGA4 & 2 & 1.39 & I & Integrin, alpha 4 \\
\hline MEIS1 & 2 & 1.42 & $\mathrm{P}$ and $\mathrm{I}$ & Meis homeobox 1 \\
\hline$N E D D 4 L$ & 2 & 1.40 & $\mathrm{P}$ & $\begin{array}{l}\text { Neural precursor cell expressed, } \\
\text { developmentally downregulated 4-like }\end{array}$ \\
\hline$N F 1$ & 2 & 1.42 & I & Neurofibromin 1 \\
\hline$N K X 2-1$ & 2 & 1.36 & $\mathrm{P}$ and $\mathrm{I}$ & NK2 homeobox 1 \\
\hline$N K X 2-2$ & 2 & 1.55 & $\mathrm{P}$ and $\mathrm{I}$ & NK2 homeobox 2 \\
\hline
\end{tabular}


Table IV. Continued.

\begin{tabular}{|c|c|c|c|c|}
\hline Gene & $\begin{array}{c}\text { Changed } \\
\text { probes }\end{array}$ & $\begin{array}{c}\text { Average } \\
\text { ratio }\end{array}$ & Position & Gene description \\
\hline NOTCH1 & 2 & 1.40 & I & Notch homolog 1, translocation-associated \\
\hline$N P Y$ & 2 & 1.33 & P and I & Neuropeptide Y \\
\hline$N R 2 F 2$ & 2 & 1.47 & $\mathrm{P}$ & Nuclear receptor subfamily 2 \\
\hline$N R 4 A 3$ & 2 & 1.66 & $\mathrm{P}$ & Nuclear receptor subfamily 4 , \\
\hline$N R P 1$ & 2 & 1.28 & $\mathrm{P}$ and I & Neuropilin 1 \\
\hline$P 4 H B$ & 2 & 1.52 & $\mathrm{P}$ & Prolyl 4-hydroxylase \\
\hline$P A X 9$ & 2 & 1.95 & $\mathrm{P}$ & Paired box 9 \\
\hline PHOX $2 B$ & 2 & 1.68 & $\mathrm{I}$ & Paired-like homeobox $2 b$ \\
\hline POMC & 2 & 1.41 & $\mathrm{P}$ & Proopiomelanocortin \\
\hline POU $4 F 1$ & 2 & 1.42 & $\mathrm{P}$ & POU class 4 homeobox 1 \\
\hline POU4F3 & 2 & 1.60 & $\mathrm{P}$ and $\mathrm{D}$ & POU class 4 homeobox 3 \\
\hline PTGS2 & 2 & 1.56 & $\mathrm{P}$ & Prostaglandin-endoperoxide synthase 2 \\
\hline SALL1 & 2 & 1.46 & $\mathrm{P}$ & Sal-like 1 \\
\hline SIM1 & 2 & 1.33 & $\mathrm{P}$ and $\mathrm{I}$ & Single-minded homolog 1 \\
\hline SSTR1 & 2 & 1.79 & $\mathrm{I}$ & Somatostatin receptor 1 \\
\hline TCF3 & 2 & 1.28 & $\mathrm{I}$ & Transcription factor 3 \\
\hline TXNRD1 & 2 & 1.54 & $\mathrm{I}$ & Thioredoxin reductase 1 \\
\hline WIT1 & 2 & 1.79 & $\mathrm{I}$ & Wilms tumor upstream neighbor 1 \\
\hline WNT1 & 2 & 1.30 & $\mathrm{I}$ & Wingless-type MMTV integration site family \\
\hline WNT7A & 2 & 1.67 & $\mathrm{P}$ and I & Wingless-type MMTV integration site family \\
\hline
\end{tabular}

Several probes are present on the microarray for each gene. Average ratio, representing the average ratio of probe density of positive probe in cancerous to that in non-cancerous breast tissue, was calculated by SAM software. P, promoter; I, inside, D, downstream.

Hierarchical cluster display of these hypermethylated genes in the 10 cancerous and 10 non-cancerous breast samples showed 2 separate clusters; one contained all the breast cancer samples and the other contained all the noncancerous breast samples (Fig. 2A). Enrichment analysis of the GO terms of the 70 hypermethylated genes showed that these hypermethylated genes were involved in development, such as the homeobox genes, including the HOX and PAX gene clusters, and regulation, such as NOTCH1, NEUROG3, SOX9 and TCF3. The microarray raw data have been deposited in NCBI's Gene Expression Omnibus (http://www.ncbi.nlm.nih. gov/geo) and are accessible through GEO series accession number GSE33450.

Validation of the hypermethylated genes. In contrast to traditional bisulfite-modified sequencing, in which the PCR product is firstly transformed into single clones, direct bisulfitemodified sequencing directly uses the PCR product for Sanger sequencing. Even if there are high background signals, such as heterozygote double peaks at site $\mathrm{C}$ of some $\mathrm{CpG}$ islands, originating from $\mathrm{PCR}$ products containing methylated $\mathrm{C}$ and $\mathrm{T}$ converted from unmethylated $\mathrm{C}$, directing bisulfite-modified sequencing is time-effective and easily operated, and therefore has been widely used for rapidly screening methylated sites and finding methylation levels of target sequences (16). To confirm the reliability of our MIRA microarray analysis results, 4 genes were selected for validation in breast cancer and non-cancerous samples using direct bisulfite sequencing. Through direct bisulfite sequencing, widespread methylations were observed in intragenic regions of the WT1, PAX6 and
ITGA4 genes, and in the promoter region of OTX2 in breast cancer tissue. Methylations were also observed in noncancerous tissue for the OTX2 and WT1 but not for the PAX6 and ITGA4 genes (Fig. 3).

Based on direct sequencing results, we designed primers that amplify DNA fragments with the richest methylated $\mathrm{CpG}$ and further validated methylation states of the WT1, OTX2, PAX6 and ITGA4 genes using COBRA assay in 5 independent cancerous and 5 non-cancerous samples. As shown in Fig. 4, the WT1, OTX2 and PAX6 genes were confirmed to be hypermethylated in breast cancer tissues, compared to non-cancerous breast tissues. Both direct bisulfite sequencing and COBRA validation results demonstrate reliability of our MIRA microarray analysis of DNA methylation.

Relationship between methylation and gene expression. To further explore the relationship between DNA methylation and gene expression, we also analyzed gene expression profiling, with the same batch of cancerous and non-cancerous samples used in the MIRA microarray analysis, by using gene expression microarray, which is designed to detect mRNA of 22981 genes (RefSeqAccession). In our study, $62.3 \%$ of these genes were found to be expressed in breast tissues. A total of 1308 transcripts were identified as being significantly differentially expressed in breast cancer tissues relative to non-cancerous tissues (GSE33450). Cluster analysis of gene expression data of the 70 hypermethylated genes identified by MIRA microarray revealed that the relationship between methylation and gene expression was complex. As shown in Fig. 2B, expression of some hypermethylated genes (such as MMP9 and NID2) in 


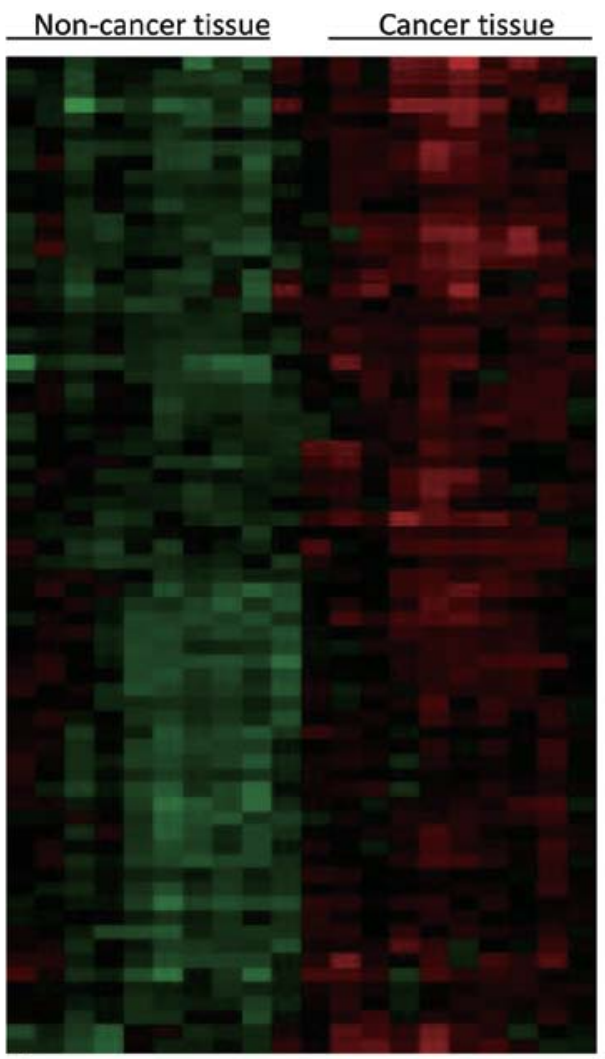

A

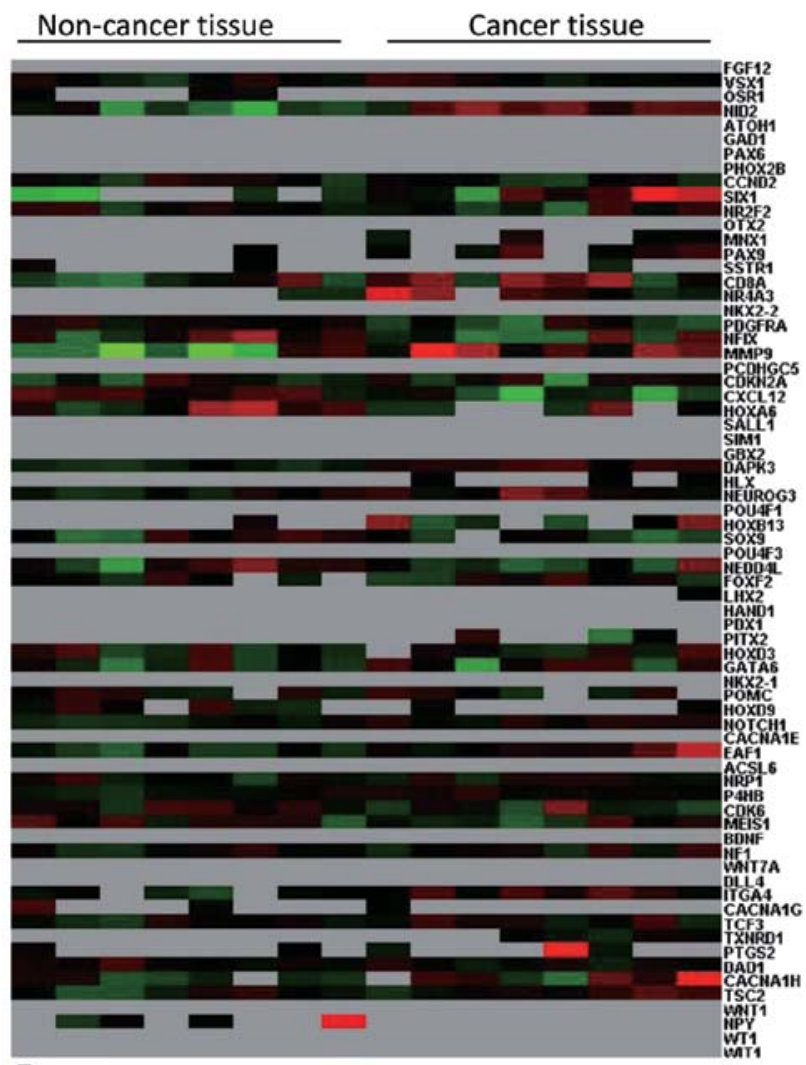

B

Figure 2. Clustering display of 70 hypermethylated genes in 10 cancerous and 10 non-cancerous breast tissues. (A) Cluster according to DNA methylation levels. (B) Cluster according to gene expression levels. In the methylation clustering heatmap, red indicates hypermethylation and green indicates hypomethylation. In the expression clustering heatmap, red indicates upregulation, green indicates downregulation and the grey bar indicates no expression. In expression profilings, $62.3 \%$ of all genes in the microarray were detected to be expressed after removing low signal probes.
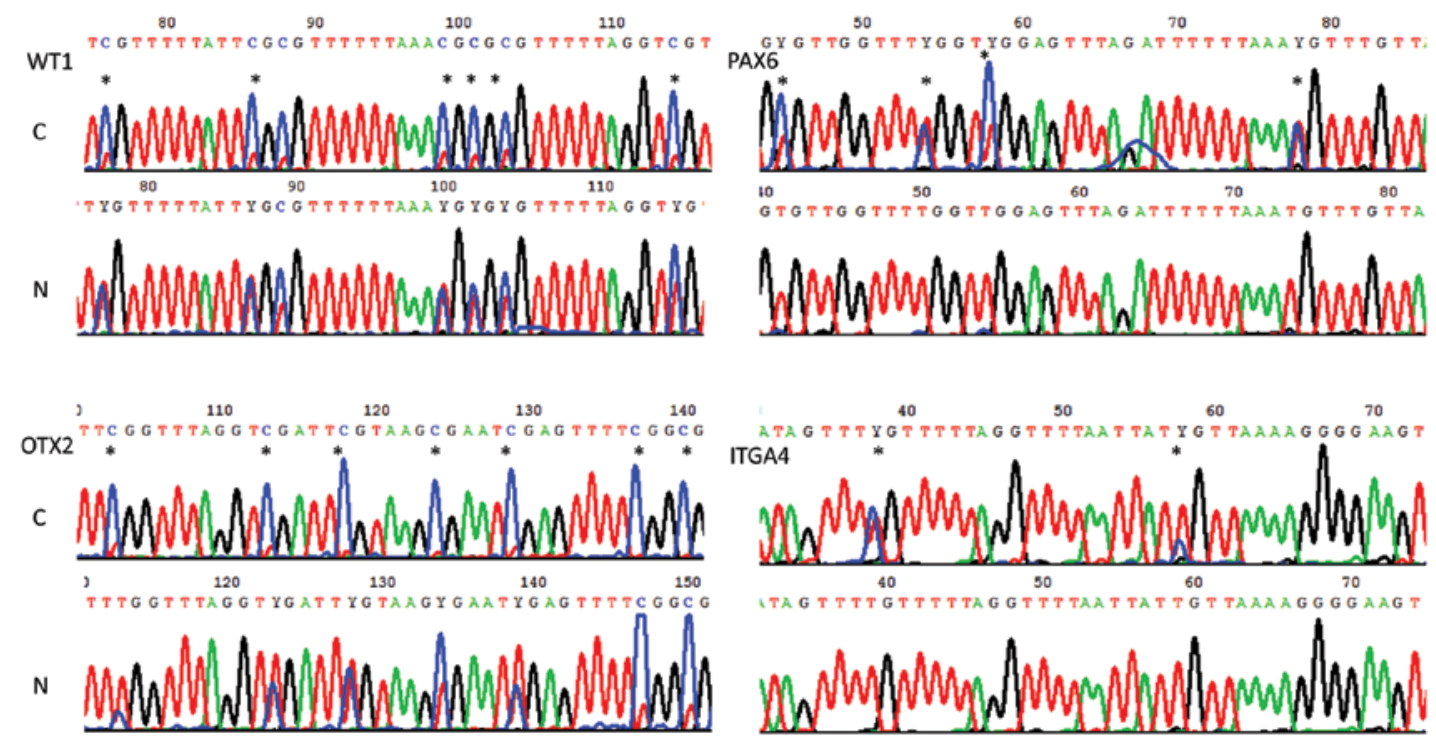

Figure 3. CpG methylations of WT1, OTX2, PAX6 and ITGA4 were measured by direct bisulfite sequencing. Hypermethylated CpGs (*) were observed at nucleotide $+262(38)$ and $+282(58)$ of the ITGA4 gene, at nucleotide $+2094(76),+2104(86),+2117(99),+2119(101),+2121(103)$ and $+2132(114)$ of the WT1 gene, at nucleotide $+6798(45),+6807(54),+6811(58)$ and $+6832(79)$ of the PAX6 gene, and at nucleotide $-3992(103),-4002(113),-4007(118),-4018(129),-4026$ (137) and -1029 (140) of positive chain of the OTX2 gene in breast cancer tissue (C), relative to non-cancerous breast tissue (N). Y indicates T or C.

cancer tissues were upregulated while expression of some other genes (such as CXCL12 and NEDD4L) were downregulated. It is worthy of note that there were many highly methylated genes with expression levels that were too low to be detected using the gene expression microarray in both cancerous and non-cancerous breast tissues, for examples, PAX6 and WT1. 


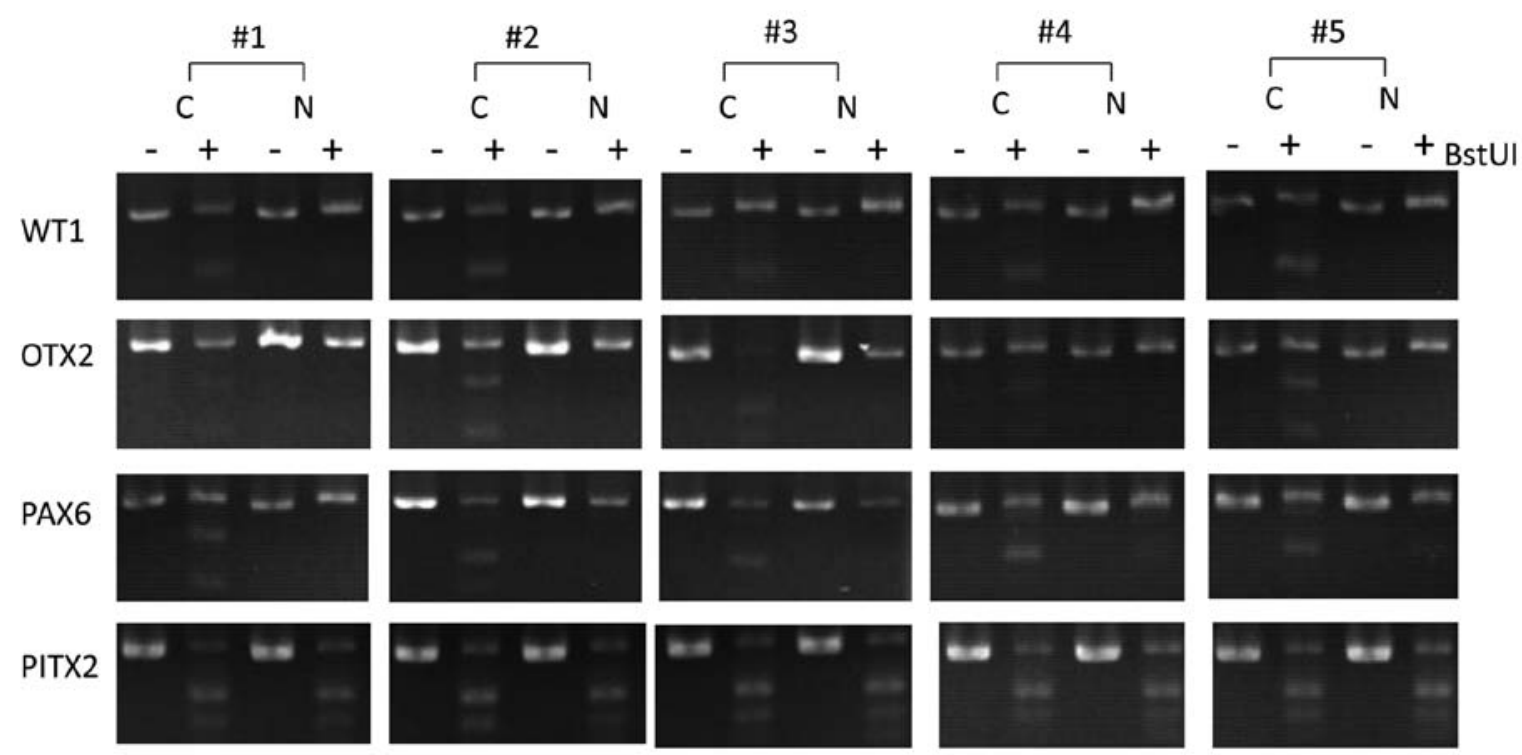

Figure 4. Methylations of the WT1, OTX2, PAX6 and PITX2 genes were validated using combined bisulfite restriction analysis (COBRA). C and N denote cancerous and non-cancerous tissue, respectively. In each case, undigested product (-) is shown next to digested product (+).

\section{N1 N2 N3 N4 N5 C1 C2 C3 C4 C5 +}

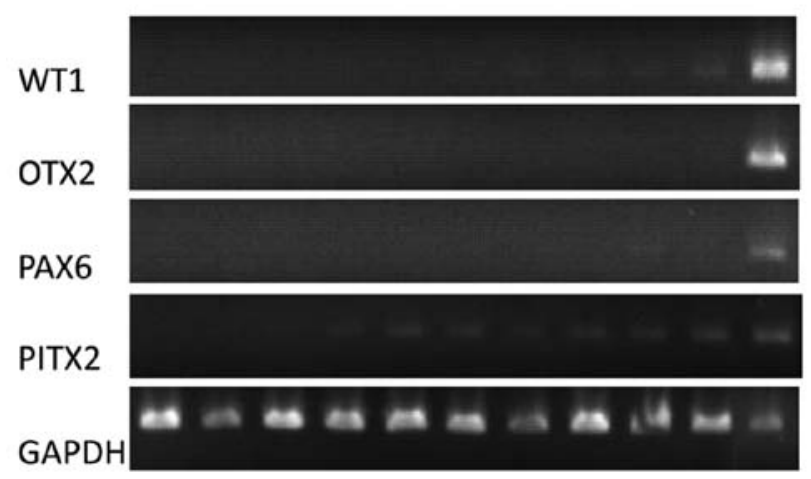

Figure 5. Low expression levels of the WT1, OTX2, PAX6 and PITX2 genes were validated in cancer breast tissue $(\mathrm{C})$ and non-cancer breast tissue $(\mathrm{N})$ using reserve transcription PCR. Positive control (+) of WT1 was total RNA from uterine tissue. Positive control of $O T X 2$ and PAX6 was total RNA from cerebellum tissue. Positive control of PITX2 was total RNA from one breast cancer tissue. Glyceraldehyde 3-phosphate dehydrogenase (GAPDH) was used as the internal control.

To confirm the gene expression microarray results, we selected the 4 hypermethylated genes WT1, PAX6, OTX2 and ITGA4, whose hypermethylation had been verified by COBRA, for gene expression detection by RT-PCR assay. The online gene expression database (www.genecards.org) showed these 4 genes are not expressed in most breast tissues. For example, SAGE data show that WT1, OTX2, PAX6 and PITX2 are weakly expressed in breast cancer tissues and are not expressed in non-cancerous tissues. Therefore, we used tissues other than breast tissue as positive controls of RT-PCR for WT1, OTX2 and PAX6. Our RT-PCR results showed that WT1 and PITX2 were only weakly expressed in the breast cancer tissues and were not expressed in most non-cancerous tissues. OTX2 and $P A X 6$ were not expressed in either cancerous or non-cancerous breast tissues (Fig. 5).

\section{Discussion}

In this study, we performed MIRA microarray analysis in 10 cancerous and 10 non-cancerous breast samples and validated methylation microarray results in another 5 cancerous and 5 non-cancerous tissues. As the screening was taken at genome-scale level, the sample size in this study was similar with the sample size in previous studies which were also performed at a genome-scale level $(10,17)$. We screened 70 hypermethylated genes in breast cancer tissues. Numerous hypermethylated genes screened in this study are homeobox genes. For example, the $4 H O X, 2 P A X$ and $2 P O U$ genes were found to be hypermethylated in breast cancer. Our results strengthen the hypothesis that multiple $\mathrm{CpG}$ islands within the HOX clusters and near other homeobox genes are frequent methylation targets in cancer $(11,17)$.

PITX2 methylation has been reported be strongly correlated with increased risk of recurrence in breast cancer $(18,19)$. In this study, we found that PITX2 was hypermethylated in breast cancer patients. However, COBRA assay showed that PITX2 was also hypermethylated in non-cancer patients, suggesting that PITX2 might not be an ideal methylation biomarker for breast cancer recurrence in Chinese populations. However, further studies are required to confirm this hypothesis.

From our MIRA-based methylation screening, a panel of novel hypermethylated genes that might hold promise as biomarkers for the early detection of breast cancer was screened, for example, WT1, OTX2, FGF12, SOX9 and MNX1. Using COBRA assay, we confirmed that $W T 1$ and $O T X 2$ were hypermethylated in independent cancerous and non-cancerous samples. WT1 has been shown to be expressed in primary breast tumors despite tumor-specific promoter methylation (20) and its promoter methylation might not silence the mRNA expression of WT1 during the development of breast cancer (21). In agreement with previous studies, $W T 1$ is weakly expressed in breast cancer tissues and is not expressed in non-cancerous tissues in this study. Contrary to previous studies, all detected 
methylated sites of the WT1 gene in our study were located at gene body. Several isoforms of WT1 have been reported to be expressed in breast tumors $(22,23)$. Perhaps, intragenic methylation of $W T 1$ is associated with alternative splicing.

We also explored the relationship between DNA methylation and gene expression. Gene expression is functionally more important than DNA methylation and it is generally recognized that gene promoter hypermethylation results in transcriptional silence. However, exceptional cases, in which gene expression levels have nothing to do with their promoter methylation status, have also been reported. For an example, the promoter of the vimentin gene has recently been found hypermethylated in colorectal cancer, while it is not expressed in colorectal tissues (8). Methylation is also common in gene body (10). However, the function of intragenic DNA methylation still remains to be defined. It has recently been shown that DNA methylation in downstream exons was only weakly linked to transcription level (24). In our results, the gene body of WT1, PAX6 and ITGA4 and the promoter of OTX2 were found hypermethylated in breast cancer tissue and their expression levels were very low in both cancerous and non-cancerous breast tissue, suggesting that intragenic DNA methylation of WT1, PAX6 and ITGA4 and the promoter methylation of OTX2 may have, if any, only a very weak function in regulating gene expression.

To display the relationship between methylation and gene expression from a large perspective, 70 hypermethylated genes in breast cancer tissues screened by MIRA microarray analysis were clustered based on their gene expression levels. Our data showed most of the hypermethylated genes in cancer are not expressed in either cancerous or non-cancerous tissues. Few studies have paid attention to this phenomenon before. To validate it, we selected 4 hypermethylated genes that are not expressed in either cancerous and non-cancerous tissues during tumorigenesis and verified their methylation status and expression levels using COBRA and RT-PCR, respectively.

Gene expression microarray based strategy has been widely used for screening DNA methylation biomarkers on a genomic scale. This strategy is based on the recognition that gene promoter hypermethylation results in transcriptional silence. Based on upregulated genes after demethylating agent treatment, hypermethylated genes could be detected. In this study, intragenic DNA methylation of WT1, PAX6 and ITGA4 and the promoter methylation of OTX2 did not show tight association with gene expressions. Their methylations would not have been detected, if gene expression based strategy had been used. Different from gene expression microarray-based strategy, the MIRA approach directly detects methylated genes at the DNA level. It can effectively screen methylated genes irrespective of whether it expresses mRNA or not. Therefore, we can screen more candidate methylated genes for further clinical validation using this tool than the gene expression microarray-based strategy.

To date, no single gene has been found to be methylated in all types of breast cancer. Methylation signature, consisting of a panel of genes, has been considered a research strategy of biomarker for the early detection of breast cancer. Currently, most studies select genes based on the methylation state in other cancer modes or known gene function, including regulating function of methylation on gene expressions. These genes are mostly tumor suppressor genes, which are hypermethylated in promoter and highly expressed in non-cancerous tissue. Our results expand our knowledge of hypermethylated genes and methylation sites in breast cancer and provide further options other than promoter methylation of few tumor suppressor genes to study methylation biomarkers of breast cancer detection, including both single gene methylation and methylation signature biomarkers. Next, we will further assess the potential of these hypermethylated genes for their use in the early detection of breast cancer in well-defined populations, using accessible samples, for example, nipple aspirate fluid.

In conclusion, we detected 70 genes that were significantly hypermethylated in promoter and/or gene body in breast cancer tissues, and most of them were lowly expressed at the RNA level in both cancerous and non-cancerous breast tissue. These results expand our knowledge of hypermethylated genes and methylation sites, deepen our understanding of the relationship between methylation and gene expression and provide a fundamental basis to further validate the feasibility of discovered methylation biomarkers used for the early detection of breast cancer in larger samples in the future. In addition, we can select more candidate methylated genes for clinical validation using the MIRA approach than using the gene expression microarray-based strategy.

\section{Acknowledgements}

This work was supported by the Guangdong Natural Science Foundation Grant No. S2011010003747. We thank Dr Liang Zhang and BioChain (Beijing) Science \& Technology Inc. for their technical assistance.

\section{References}

1. Ferlay J, Shin HR, Bray F, Forman D, Mathers C and Parkin DM: Estimates of worldwide burden of cancer in 2008: GLOBOCAN 2008. Int J Cancer 127: 2893-2917, 2010.

2. Esteller M: Epigenetics in cancer. N Engl J Med 358: 1148-1159, 2008.

3. Martens JW, Margossian AL, Schmitt M, Foekens J and Harbeck N: DNA methylation as a biomarker in breast cancer. Future Oncol 5: 1245-1256, 2009.

4. Jovanovic J, Ronneberg JA, Tost J and Kristensen V: The epigenetics of breast cancer. Mol Oncol 4: 242-254, 2010.

5. Brooks J, Cairns P and Zeleniuch-Jacquotte A: Promoter methylation and the detection of breast cancer. Cancer Causes Control 20: 1539-1550, 2009.

6. Sproul D, Nestor C, Culley J, et al: Transcriptionally repressed genes become aberrantly methylated and distinguish tumors of different lineages in breast cancer. Proc Natl Acad Sci USA 108: 4364-4369, 2011

7. Ngan CY, Yamamoto H, Seshimo I, et al: Quantitative evaluation of vimentin expression in tumour stroma of colorectal cancer. $\mathrm{Br}$ J Cancer 96: 986-992, 2007.

8. Chen WD, Han ZJ, Skoletsky J, et al: Detection in fecal DNA of colon cancer-specific methylation of the nonexpressed vimentin gene. J Natl Cancer Inst 97: 1124-1132, 2005.

9. Ruike Y, Imanaka Y, Sato F, Shimizu K and Tsujimoto G: Genome-wide analysis of aberrant methylation in human breast cancer cells using methyl-DNA immunoprecipitation combined with high-throughput sequencing. BMC Genomics 11: 137, 2010.

10. Wu X, Rauch TA, Zhong X, et al: $\mathrm{CpG}$ island hypermethylation in human astrocytomas. Cancer Res 70: 2718-2727, 2010.

11. Tommasi S, Karm DL, Wu X, Yen Y and Pfeifer GP: Methylation of homeobox genes is a frequent and early epigenetic event in breast cancer. Breast Cancer Res 11: R14, 2009.

12. Rauch TA and Pfeifer GP: The MIRA method for DNA methylation analysis. Methods Mol Biol 507: 65-75, 2009. 
13. Richards AA, Santos LJ, Nichols HA, et al: Cryptic chromosomal abnormalities identified in children with congenital heart disease. Pediatr Res 64: 358-363, 2008.

14. Leung TY, Vogel I, Lau TK, et al: Identification of submicroscopic chromosomal aberrations in fetuses with increased nuchal translucency and apparently normal karyotype. Ultrasound Obstet Gynecol 38: 314-319, 2011.

15. Xiong Z and Laird PW: COBRA: a sensitive and quantitative DNA methylation assay. Nucleic Acids Res 25: 2532-2534, 1997.

16. Ibanez de Caceres I, Cortes-Sempere M, Moratilla C, et al IGFBP-3 hypermethylation-derived deficiency mediates cisplatin resistance in non-small-cell lung cancer. Oncogene 29 : $1681-1690,2010$

17. Rauch T, Wang Z, Zhang X, et al: Homeobox gene methylation in lung cancer studied by genome-wide analysis with a microarraybased methylated CpG island recovery assay. Proc Natl Acad Sci USA 104: 5527-5532, 2007.

18. Maier S, Nimmrich I, Koenig T, et al: DNA-methylation of the homeodomain transcription factor PITX2 reliably predicts risk of distant disease recurrence in tamoxifen-treated, node-negative breast cancer patients - Technical and clinical validation in a multi-centre setting in collaboration with the European Organisation for Research and Treatment of Cancer (EORTC) PathoBiology group. Eur J Cancer 43: 1679-1686, 2007.
19. Harbeck N, Nimmrich I, Hartmann A, et al: Multicenter study using paraffin-embedded tumor tissue testing PITX2 DNA methylation as a marker for outcome prediction in tamoxifentreated, node-negative breast cancer patients. J Clin Oncol 26: 5036-5042, 2008 .

20. Loeb DM, Evron E, Patel CB, et al: Wilms' tumor suppressor gene (WT1) is expressed in primary breast tumors despite tumorspecific promoter methylation. Cancer Res 61: 921-925, 2001

21. Yang JL, Klinkebiel D, Boland MJ, Tang L and Christman JK: [Promoter methylation and mRNA expression of WT1 gene in MCF10 breast cancer model]. Zhonghua Bing Li Xue Za Zhi 36: 253-258, 2007 (In Chinese).

22. Silberstein GB, Van Horn K, Strickland P, Roberts CT Jr and Daniel CW: Altered expression of the WT1 Wilms tumor suppressor gene in human breast cancer. Proc Natl Acad Sci USA 94: 8132-8137, 1997.

23. Laux DE, Curran EM, Welshons WV, Lubahn DB and Huang TH: Hypermethylation of the Wilms' tumor suppressor gene $\mathrm{CpG}$ island in human breast carcinomas. Breast Cancer Res Treat 56: 35-43, 1999.

24. Brenet F, Moh M, Funk P, et al: DNA methylation of the first exon is tightly linked to transcriptional silencing. PLoS One 6: e14524, 2011. 